\title{
The combined Laplace-homotopy analysis method for partial differential equations
}

\author{
Javad Vahidi \\ Department of Mathematics, Iran University of Science and Technology, Tehran, Iran.
}

\begin{abstract}
In this paper, the Laplace transform homotopy analysis method (LHAM) is employed to obtain approximate analytical solutions of the linear and nonlinear differential equations. This method is a combined form of the Laplace transform method and the homotopy analysis method. The proposed scheme finds the solutions without any discretization or restrictive assumptions and is free from round-off errors and therefore, reduces the numerical computations to a great extent. Some illustrative examples are presented and the numerical results show that the solutions of the LHAM are in good agreement with those obtained by exact solution. (c)2016 All rights reserved.
\end{abstract}

Keywords: Homotopy analysis method, Laplace transform method, partial differential equation. 2010 MSC: 54A40, 26E50.

\section{Introduction}

Nonlinear phenomena have important effects on applied mathematics, physics and issues related to engineering; many such physical phenomena are modeled in terms of nonlinear partial differential equations. The importance of obtaining the exact or approximate solutions of linear and nonlinear partial differential equations in physics and mathematics is still a significant problem that needs new methods to discover exact or approximate solutions. Most new linear and nonlinear equations do not have a precise analytic solution; so, numerical methods have largely been used to handle these equations. There are also analytic techniques for nonlinear equations. Some of the classic analytic methods are Lyapunovs artificial small parameter method [1], perturbation techniques [3, 4], $\delta$ expansion method [5] and Hirota bilinear method [6, 7]. In recent years many authors have paid attention to studying the solutions of linear and nonlinear partial differential equations by using various methods. Among these are the Adomian decomposition method (ADM) [8], Hes semi-inverse

Email address: jvahidi@iust.ac.ir (Javad Vahidi) 
method [9], the tanh method, the homotopy perturbation method (HPM), the sinhcosh method, the differential transform method and the variational iteration method (VIM) [10, 11, 12, 13, 14, 15, 16, 17, 18, 19. Most of these methods have their inbuilt deficiencies like the calculation of Adomians polynomials, the Lagrange multiplier, divergent results and huge computational work. In 1992, Liao [20] employed the basic ideas of the homotopy in topology to propose a general analytic method for nonlinear problems, namely the Homotopy analysis method (HAM), [16, 19, 21, 22]. In recent years, homotopy analysis method has been used in obtaining approximate solutions of a wide class of differential, integral and integro-differential equations. The method provides the solution in a rapidly convergent series with components that are elegantly computed. The Laplace transform is totally incapable of handling nonlinear equations because of the difficulties that are caused by the nonlinear terms. Various ways have been proposed recently to deal with these nonlinearities such as the Adomian decomposition method [23] and the Laplace decomposition algorithm [24, 25, 26, [27, 29]. Inspired and motivated by the ongoing research in this area, we use the homotopy analysis method coupled with the Laplace transformation for solving the linear and nonlinear equations in this paper. It is worth mentioning that the proposed method is an elegant combination of the Laplace transformation and the homotopy analysis method. This paper considers the effectiveness of the Laplace homotopy analysis method in solving linear and nonlinear partial differential equations, both homogeneous and non-homogeneous.

\section{Basic idea of LHAM}

To illustrate the basic idea of this method, we consider the general form of two-dimensional non-homogeneous partial differential equations with a variable coefficient of the form

$$
\frac{\partial u}{\partial t}=\mu(x) \frac{\partial^{2} u}{\partial x^{2}}+\varphi(x, t)
$$

subject to the boundary conditions

$$
u(0, t)=g_{0}(t), \quad u(1, t)=g_{1}(t),
$$

and the initial condition

$$
u(x, 0)=f(x) .
$$

The methodology consists of applying a Laplace transform on both sides of Eqs. 2.1), 2.2 and in view of the initial condition, we get

$$
\begin{aligned}
& \frac{\partial^{2} \bar{u}}{\partial x^{2}}-\frac{s \bar{u}}{\mu x}+\frac{L[\phi(x, t)]+f(x)}{\mu x}=0, \\
& \bar{u}(0, t)=\bar{g}_{0}(t), \quad \bar{u}(1, t)=\bar{g}_{1}(t),
\end{aligned}
$$

which is a second-order boundary value problem. By means of generalizing the traditional homotopy method, Liao [22] constructed the so-called zero-order deformation equation

$$
(1-p) \mathcal{L}\left[\phi(x, s ; p)-\bar{u}_{0}(x, s)\right]=p \hbar \mathcal{H}(x, s) \mathcal{N}[\phi(x, s ; p)]
$$

where $p \in[0,1]$ is the embedding parameter, $h \neq 0$ is a non-zero auxiliary parameter, $\mathcal{H}(x, s) \neq 0$ is an auxiliary function, $\bar{u}_{0}(x, s)$ is an initial guess of $\bar{u}(x, s)$ and $\phi(x, s ; p)$ is an unknown function and $\mathcal{L}$ an auxiliary linear operator with the property

$$
\mathcal{L}[\phi(x, s ; p)]=\frac{\partial^{2} \phi(x, s ; p)}{\partial x^{2}} .
$$


We define a nonlinear operator $\mathcal{N}$ as

$$
\mathcal{N}[\phi(x, s ; p)]=\frac{\partial^{2} \phi(x, s ; p)}{\partial x^{2}}-\frac{s \phi(x, s ; p)}{\mu(x)}+\frac{L[\varphi(x, t)]+f(x)}{\mu(x)} .
$$

It is important, that one has great freedom to choose auxiliary things in LHAM. Obviously, when $p=0$ and $p=1$, it holds

$$
\phi(x, s ; 0)=\bar{u}_{0}(x, s), \quad \phi(x, s ; 1)=\bar{u}(x, s),
$$

respectively. Thus, as $p$ increases from 0 to 1 , the solution $\phi(x, s ; p)$ varies from the initial guess $\bar{u}_{0}(x, s)$ to the solution $\bar{u}(x, s)$. Expanding $\phi(x, s ; p)$ in Taylor series with respect to $p$, we have

$$
\phi(x, s ; p)=\bar{u}_{0}(x, s)+\sum_{m=1}^{+\infty} \bar{u}_{m}(x, s) p^{m},
$$

where

$$
\bar{u}_{m}(x, s)=\left[\frac{1}{m !} \frac{\partial^{m} \phi(x, s ; p)}{\partial p^{m}}\right]_{p=0} .
$$

If the auxiliary linear operator, the initial guess, the auxiliary parameter $\hbar$, and the auxiliary function are so properly chosen, the series 2.10 converges at $p=1$, then we have

$$
\bar{u}(x, s)=\bar{u}_{0}(x, s)+\sum_{m=1}^{+\infty} \bar{u}_{m}(x, s),
$$

which must be one of solutions of original nonlinear equation, as proved by [22]. As $\hbar=-1$ and $\mathcal{H}(x, s)=1$, Eq. 2.5 becomes

$$
(1-p) \mathcal{L}\left[\phi(x, s ; p)-\bar{u}_{0}(x, s)\right]+p \mathcal{N}[\phi(x, s ; p)]=0,
$$

which is used mostly in the homotopy perturbation method [3], where as the solution obtained directly, without using Taylor series. Define the vector

$$
\overrightarrow{\vec{u}}_{n}=\left\{\bar{u}_{0}(x, s), \bar{u}_{1}(x, s), \cdots, \bar{u}_{n}(x, s)\right\} .
$$

Differentiating equation (2.5) $m$ times with respect to the embedding parameter $p$ and then setting $p=0$ and finally dividing them by $m$ !, we have the so-called $m$ th-order deformation equation

$$
\mathcal{L}\left[\bar{u}_{m}(x, s)-\chi_{m} \bar{u}_{m-1}(x, s)\right]=\hbar \mathcal{H}(x, s) \mathcal{R}_{m}\left(\vec{u}_{m-1}\right),
$$

where

$$
\mathcal{R}_{m}\left(\vec{u}_{m-1}\right)=\left[\frac{1}{(m-1) !} \frac{\partial^{m-1} \mathcal{N}[\phi(x, s ; p)]}{\partial p^{m-1}}\right]_{p=0}
$$

and

$$
\chi_{m}= \begin{cases}0, & m \leq 1 \\ 1, & m>1\end{cases}
$$

It should be emphasized that $\bar{u}_{m}(x, s)$ for $m \geq 1$ is governed by the linear equation (2.13) under the linear boundary conditions that come from original problem, which can be easily solved by symbolic computation software such as Matlab. Taking the inverse Laplace transform from $\bar{u}_{m}(x, s)$ we obtain components $u_{m}(x, s)$ of series solution of Eq. 2.1.

\section{Applications}

In order to assess the advantages and the accuracy of Laplace-homotopy analysis method for solving partial differential equations, we will consider the following three examples. 
Example 3.1. Consider the following homogeneous problem

$$
\begin{aligned}
& \frac{\partial u}{\partial t}+u \frac{\partial u}{\partial x}=0, \\
& u(x, 0)=-x .
\end{aligned}
$$

\section{LHAM approach}

By applying a Laplace transform on both sides of Eq. (3.1) we get

$$
u(x, s)=-\frac{x}{s}-\frac{1}{s} L\left[u \frac{\partial u}{\partial x}\right] .
$$

Now, we solve Eq. (3.2) by means of HAM. Therefore, we choose the linear oprator

$$
\mathcal{L}[\phi(x, s ; p)]=\phi(x, s ; p) .
$$

We define a nonlinear operator as

$$
\mathcal{N}[\phi(x, s ; p)]=\phi(x, s ; p)+\frac{x}{s}+\frac{1}{s} L\left[\phi(x, t ; p) \frac{\partial \phi(x, t ; p)}{\partial x}\right] .
$$

Using the definition (3.3) and (3.4), we construct the zeroth-order deformation equation

$$
(1-p) \mathcal{L}\left[\phi(x, s ; p)-\bar{u}_{0}(x, s)\right]=p \hbar \mathcal{H}(x, s) \mathcal{N}[\phi(x, s ; p)] .
$$

Thus, we obtain the $m$ th-order $(m \geq 1)$ deformation equation

$$
\mathcal{L}\left[\bar{u}_{m}(x, s)-\chi_{m} \bar{u}_{m-1}(x, s)\right]=\hbar \mathcal{H}(x, s) \mathcal{R}_{m}\left(\overrightarrow{\bar{u}}_{m-1}\right),
$$

where

$$
\mathcal{R}_{m}\left(\bar{u}_{m-1}\right) \stackrel{\vec{\prime}}{=} \bar{u}_{m-1}-\frac{1}{s} L\left[\sum_{i=0}^{m-1} u_{i} \frac{\partial u_{m-1-i}}{\partial x}\right]+\left(1-\chi_{m}\right)\left(\frac{x}{s}\right) .
$$

Now, the solution of the $m$ th-order $(m \geq 1)$ deformation equation $(3.5)$ is as follows

$$
\bar{u}_{m}(x, s)=\chi_{m} \bar{u}_{m-1}(x, s)+\hbar \mathcal{H}(\xi, s) \mathcal{R}_{m}\left(\overrightarrow{\bar{u}}_{m-1}(\xi, s)\right) .
$$

The initial approximation $\bar{u}_{0}(x, s)$ can be freely chosen, here we set

$$
\bar{u}_{0}(x, s)=-\frac{x}{s} .
$$

By choosing $H(x, s)=1$, the components of the LHAM solution for Eq. (3.2) are derived as follows

$$
\begin{aligned}
& \bar{u}_{1}(x, s)=-\frac{1 !}{s^{2}} x \\
& \bar{u}_{2}(x, s)=-\frac{2 !}{s^{3}} x \\
& \bar{u}_{3}(x, s)=-\frac{3 !}{s^{3}} x \\
& \bar{u}_{4}(x, s)=-\frac{4 !}{s^{3}} x
\end{aligned}
$$

In the same manner, the rest of components were obtained using the Matlab package. 
Taking the inverse Laplace of components yields

$$
\begin{aligned}
& u_{0}(x, s)=-x, \\
& u_{1}(x, s)=-x t, \\
& u_{2}(x, s)=-x t^{2}, \\
& u_{3}(x, s)=-x t^{3}, \\
& u_{4}(x, s)=-x t^{4},
\end{aligned}
$$

so that the solution $u(x, t)$ is given by

$$
u(x, t)=-x\left(1+t+t^{2}+t^{3}+\cdots\right)
$$

in series form, and

$$
u(x, t)=\frac{x}{t-1}
$$

is in closed form. That is the exact solution of Eq. (3.1).

Example 3.2. Let us consider the one dimensional non-homogeneous problem

$$
\frac{\partial u}{\partial t}+\frac{\partial u}{\partial x^{2}}+u-e^{-x}(1+2 t)=0
$$

subject to boundary conditions

$$
u(0, t)=0, \quad u_{x}(0, t)=e^{-t}-t,
$$

and the initial condition $u(x, 0)=x$ that is easily seen to have the exact solution $u(x, t)=t e^{-x}+x e^{-t}$.

\section{HAM approach}

To solve Eq. (3.8) by means of HAM, we choose the linear operator

$$
\mathcal{L}[\phi(x, t ; p)]=\frac{\partial \phi(x, t ; p)}{\partial t} .
$$

Furthermore, Eq. (3.8) suggests to define the nonlinear operator

$$
\mathcal{N}[\phi(x, t ; p)]=\frac{\partial \phi(x, t ; p)}{\partial t}+\frac{\partial^{2} \phi(x, t ; p)}{\partial x^{2}}+\phi(x, t ; p)-e^{-x}(1+2 t) .
$$

Using above definition, we construct the zeroth-order deformation equation

$$
(1-p) \mathcal{L}\left[\phi(x, t ; p)-u_{0}(x, t)\right]=p \hbar \mathcal{H}(x, t) \mathcal{N}[\phi(x, t ; p)] .
$$

According to Eqs. (3.9), (3.10), we gain the $m$ th-order $(m \geq 1)$ deformation equation

$$
\mathcal{L}\left[u_{m}(x, t)-\chi_{m} u_{m-1}(x, t)\right]=\hbar \mathcal{H}(x, t) \mathcal{R}_{m}\left(\vec{u}_{m-1}\right),
$$

where

$$
\mathcal{R}_{m}\left(\vec{u}_{m-1}\right)=\frac{\partial u_{m-1}}{\partial t}+\frac{\partial^{2} u_{m-1}}{\partial x^{2}}+u_{m-1}-\left(1-\chi_{m}\right) e^{-x}(1+2 t) .
$$


Now, the components of solution of Eq. (3.8) for $(m \geq 1)$ becomes

$$
u_{m}(x, t)=\chi_{m} u_{m-1}(x, t)+\hbar \int_{0}^{t} \mathcal{H}(x, \xi) \mathcal{R}_{m}\left(\vec{u}_{m-1}(x, \xi)\right) d \xi
$$

The initial approximation $u_{0}(x, t)$ can be freely chosen, here we set

$$
u_{0}(x, t)=x .
$$

Then, the solution of Eq. (3.8) in series form is given by

$$
u(x, t)=u_{0}(x, t)+u_{1}(x, t)+\cdots .
$$

\section{LHAM approach}

By applying a Laplace transform on both sides of Eqs. (3.8), (3.9) and in view of the initial condition, we get

$$
\begin{gathered}
\frac{\partial^{2} \bar{u}}{\partial x^{2}}+(s+1) \bar{u}-x-e^{-x}\left(\frac{1}{s}+\frac{1}{s^{2}}\right)=0 \\
\bar{u}(0, s)=\frac{1}{s^{2}}, \quad \bar{u}_{x}(0, s)=-\frac{1}{s^{2}}+\frac{1}{s+1} .
\end{gathered}
$$

Now, we solve Eq. (3.12) by means of HAM. Therefore, we choose the linear oprator

$$
\mathcal{L}[\phi(x, s ; p)]=\frac{\partial^{2} \phi(x, s ; p)}{\partial x^{2}}
$$

We define a nonlinear operator as

$$
\mathcal{N}[\phi(x, s ; p)]=\frac{\partial^{2} \phi(x, s ; p)}{\partial x^{2}}+(s+1) \phi(x, s ; p)-x-e^{-x}\left(\frac{1}{s}+\frac{1}{s^{2}}\right) .
$$

Using the definition (3.14) and (3.15), we construct the zeroth-order deformation equation

$$
(1-p) \mathcal{L}\left[\phi(x, s ; p)-\bar{u}_{0}(x, s)\right]=p \hbar \mathcal{H}(x, s) \mathcal{N}[\phi(x, s ; p)] .
$$

Thus, we obtain the $m$ th-order $(m \geq 1)$ deformation equation

$$
\mathcal{L}\left[\bar{u}_{m}(x, s)-\chi_{m} \bar{u}_{m-1}(x, s)\right]=\hbar \mathcal{H}(x, s) \mathcal{R}_{m}\left(\overrightarrow{\bar{u}}_{m-1}\right),
$$

where

$$
\mathcal{R}_{m}\left(\overrightarrow{\bar{u}}_{m-1}\right)=\frac{\partial^{2} \bar{u}_{m-1}}{\partial x^{2}}+(s+1) \bar{u}_{m-1}-\left(1-\chi_{m}\right)\left(x+e^{-x}\left(\frac{1}{s}+\frac{1}{s^{2}}\right)\right) .
$$

Now, the solution of the $m$ th-order $(m \geq 1)$ deformation equation (3.16) is as follows

$$
\bar{u}_{m}(x, s)=\chi_{m} \bar{u}_{m-1}(x, s)+\hbar \int_{0}^{x} \int_{0}^{x} \mathcal{H}(\xi, s) \mathcal{R}_{m}\left(\overrightarrow{\bar{u}}_{m-1}(\xi, s)\right) d \xi d \xi .
$$

The initial approximation $\bar{u}_{0}(x, s)$ can be freely chosen, here we set

$$
\bar{u}_{0}(x, s)=\frac{1-x}{s^{2}}+\frac{x}{1+s},
$$


which satisfies the boundary conditions (3.13). By choosing $H(x, s)=1$, the components of the LHAM solution for Eq. (3.12) are derived as follows

$$
\begin{aligned}
\bar{u}_{1}(x, s)= & \frac{-\hbar}{6}\left(\frac{-6 s-12+6 x s+12 x-3 x^{2}-3 x^{2} s+x^{3}+x^{3} s+6 s e^{-x}+12 e^{-x}}{s^{2}}\right), \\
\bar{u}_{2}(x, s)= & \frac{-\hbar^{2}}{120}\left(\frac{x^{5}-5 x^{4}+60 x^{3}-180 x^{2}+480 x-480+480 e^{-x}}{s^{2}}\right) \\
& -\frac{\hbar}{120}\left(\frac{20 x^{3}-60 x^{2}+240 x-240+240 e^{-x}}{s^{2}}\right) \\
& -\frac{\hbar}{120}\left(\frac{20 x^{3}-60 x^{2}+240 x-240+240 e^{-x}}{s^{2}}\right) \\
& -\frac{\hbar^{2}}{120}\left(\frac{2 x^{5}-10 x^{4}+80 x^{3}-240 x^{2}+480 x+480+480 e^{-x}}{s}\right) \\
& -\frac{\hbar}{120}\left(\frac{20 x^{3}-60 x^{2}+240 x-240+240 e^{-x}}{s}\right) \\
& -\frac{\hbar^{2}}{120}\left(x^{5}-5 x^{4}+20 x^{3}-60 x^{2}+120 x-120+120 e^{-x}\right)
\end{aligned}
$$

In the same manner, the rest of components were obtained using the Matlab package.

Taking the inverse Laplace of components yields

$$
\begin{aligned}
u_{1}(x, t)= & x e^{-t}+(1-x) t, \\
u_{2}(x, t)= & \frac{h}{6}\left(-x^{3}+3 x^{2}-12 x+12-12 e^{-x}\right) \\
& +\frac{h}{6}\left(-x^{3}+3 x^{2}-6 x+6-6 e^{-x}\right)
\end{aligned}
$$

Finally, the $M$ th-order approximation solution of Eq. (3.8) is as follows

$$
S_{M}=\sum_{k=0}^{M} u_{k}(x, t),
$$

Note. The series (3.11) contain the auxiliary parameter $\hbar$. The validity of the method is based on such an assumption that the series 2.10 converges at $p=1$. It is the auxiliary parameter $\hbar$ which ensures that this assumption can be satisfied. As pointed out by Liao [20], in general, by means of the so-called $\hbar$-curve, it is straightforward to choose a proper value of $\hbar$ which ensures that the solution series is convergent. In this way, we choose several valid $\hbar$ in Table 1 . Table 1 describes the analytic 3rd-order approximation solution of Eq. (3.8) by the LHAM with $\hbar=1$ and the analytic 8th-order approximation solution of Eq. (3.8) by the HAM with different value of $\hbar$ when $x=0.1$ and $1 \leq t \leq 7$. Also, Table 1 shows that, unlike the LHAM the absolute error of the HAM is dramatically increased as the time value $t$ increases, so the HAM solution validity range is restricted to a short region $(t<2)$. But the validity range can be increased by increasing the terms of the solution to more than 8 terms. On the other hand, results of the LHAM are in good agreement with 
those obtained by exact solution. The LHAM solution is almost valid for a large wide range of times that shows the present method can solve a non-homogeneous parabolic equation with a high degree of accuracy by the three first terms only.

Table 1: The absolute error of LHAM and HAM with $x=0.1$

\begin{tabular}{cccc}
\hline$t$ & $L H A M(\hbar=-1)$ & $H A M(\hbar=-0.5)$ & $H A M(\hbar=-1)$ \\
\hline 1 & $4.1579 \times 10^{-9}$ & $6.6293 \times 10^{-4}$ & $6.3808 \times 10^{-4}$ \\
2 & $5.4779 \times 10^{-9}$ & $2.1794 \times 10^{-3}$ & $3.2671 \times 10^{-1}$ \\
3 & $6.8465 \times 10^{-9}$ & $5.2384 \times 10^{-3}$ & $1.2560 \times 10^{+1}$ \\
4 & $8.2158 \times 10^{-9}$ & $3.1065 \times 10^{-3}$ & $1.6728 \times 10^{+2}$ \\
5 & $9.5850 \times 10^{-9}$ & $5.5076 \times 10^{-2}$ & $1.2464 \times 10^{+3}$ \\
6 & $1.0954 \times 10^{-8}$ & $8.7898 \times 10^{-3}$ & $6.4312 \times 10^{+3}$ \\
7 & $1.2324 \times 10^{-8}$ & $4.4034 \times 10^{-1}$ & $2.5753 \times 10^{+4}$ \\
\hline
\end{tabular}

Example 3.3. Let us consider the one dimensional non-homogeneous problem

$$
\frac{\partial^{2} u}{\partial t^{2}}-\frac{\partial^{2} u}{\partial x^{2}}+u=0
$$

subject to boundary conditions

$$
u(0, t)=\cosh t, \quad u_{x}(0, t)=1,
$$

and the initial conditions

$$
u(0, t)=\sin x+1, \quad u_{t}(x, 0)=0,
$$

that is easily seen to have the exact solution $u(x, t)=\sin x+\cosh t$.

\section{HAM approach}

To solve Eq. (3.17) by means of HAM, we choose the linear operator

$$
\mathcal{L}[\phi(x, t ; p)]=\frac{\partial^{2} \phi(x, t ; p)}{\partial t^{2}}
$$

Furthermore, Eq. (3.17) suggests to define the nonlinear operator

$$
\mathcal{N}[\phi(x, t ; p)]=\frac{\partial^{2} \phi(x, t ; p)}{\partial t^{2}}+\frac{\partial^{2} \phi(x, t ; p)}{\partial x^{2}}+\phi(x, t ; p) .
$$

Using above definition, we construct the zeroth-order deformation equation

$$
(1-p) \mathcal{L}\left[\phi(x, t ; p)-u_{0}(x, t)\right]=p \hbar \mathcal{H}(x, t) \mathcal{N}[\phi(x, t ; p)] .
$$

According to Eqs. (3.19), 3.20), we gain the $m$ th-order $(m \geq 1)$ deformation equation

$$
\mathcal{L}\left[u_{m}(x, t)-\chi_{m} u_{m-1}(x, t)\right]=\hbar \mathcal{H}(x, t) \mathcal{R}_{m}\left(\vec{u}_{m-1}\right),
$$


where

$$
\mathcal{R}_{m}\left(\vec{u}_{m-1}\right)=\frac{\partial^{2} u_{m-1}}{\partial t^{2}}+\frac{\partial^{2} u_{m-1}}{\partial x^{2}}+u_{m-1} .
$$

Now, the components of solution of Eq. (3.17) for $(m \geq 1)$ becomes

$$
u_{m}(x, t)=\chi_{m} u_{m-1}(x, t)+\hbar \int_{0}^{t} \int_{0}^{t} \mathcal{H}(x, \xi) \mathcal{R}_{m}\left(\vec{u}_{m-1}(x, \xi)\right) d \xi d \xi
$$

The initial approximation $u_{0}(x, t)$ can be freely chosen, here we set

$$
u_{0}(x, t)=1+\sin x .
$$

Then, the solution of Eq. (3.17) in series form is given by

$$
u(x, t)=u_{0}(x, t)+u_{1}(x, t)+\cdots
$$

\section{LHAM approach}

By applying a Laplace transform on both sides of Eqs. (3.17), (3.18) and in view of the initial condition, we get

$$
\begin{gathered}
\frac{\partial^{2} \bar{u}}{\partial x^{2}}+\left(1-s^{2}\right) \bar{u}+s(1+\sin x)=0, \\
\bar{u}(0, s)=\frac{s}{s^{2}-1}, \quad \bar{u}_{x}(0, s)=\frac{1}{s} .
\end{gathered}
$$

Now, we solve Eq. (3.22) by means of HAM. Therefore, we choose the linear oprator

$$
\mathcal{L}[\phi(x, s ; p)]=\frac{\partial^{2} \phi(x, s ; p)}{\partial x^{2}} .
$$

We define a nonlinear operator as

$$
\mathcal{N}[\phi(x, s ; p)]=\frac{\partial^{2} \phi(x, s ; p)}{\partial x^{2}}+\left(1-s^{2}\right) \phi(x, s ; p)+s(1+\sin x) .
$$

Using the definition (3.24) and (3.25), we construct the zeroth-order deformation equation

$$
(1-p) \mathcal{L}\left[\phi(x, s ; p)-\bar{u}_{0}(x, s)\right]=p \hbar \mathcal{H}(x, s) \mathcal{N}[\phi(x, s ; p)] .
$$

Thus, we obtain the $m$ th-order $(m \geq 1)$ deformation equation

$$
\mathcal{L}\left[\bar{u}_{m}(x, s)-\chi_{m} \bar{u}_{m-1}(x, s)\right]=\hbar \mathcal{H}(x, s) \mathcal{R}_{m}\left(\overrightarrow{\bar{u}}_{m-1}\right)
$$

where

$$
\mathcal{R}_{m}\left(\overrightarrow{\bar{u}}_{m-1}\right)=\frac{\partial^{2} \bar{u}_{m-1}}{\partial x^{2}}+\left(1-s^{2}\right) \bar{u}_{m-1}+\left(1-\chi_{m}\right)(s(1+\sin x)) .
$$

Now, the solution of the $m$ th-order $(m \geq 1)$ deformation equation (3.26) is as follows

$$
\bar{u}_{m}(x, s)=\chi_{m} \bar{u}_{m-1}(x, s)+\hbar \int_{0}^{x} \int_{0}^{x} \mathcal{H}(\xi, s) \mathcal{R}_{m}\left(\overrightarrow{\bar{u}}_{m-1}(\xi, s)\right) d \xi d \xi .
$$

The initial approximation $\bar{u}_{0}(x, s)$ can be freely chosen, here we set

$$
\bar{u}_{0}(x, s)=\frac{s}{s^{2}-1}+\frac{x}{s},
$$


which satisfies the boundary conditions $(3.23)$. By choose $H(x, s)=1$, the components of the LHAM solution for Eq. $(3.22)$ are derived as follows

$$
\begin{aligned}
\bar{u}_{1}(x, s)= & \frac{-\hbar}{6}\left(\frac{s^{2} x^{3}-6 s^{2} x+6 s^{2} \sin x-x^{3}}{s}\right) \\
\bar{u}_{2}(x, s)= & \frac{\hbar^{2}}{120}\left(x^{5}-20 x^{3}+120 x-120 \sin x\right) \\
& +\frac{\hbar}{120}\left(-2 \hbar x^{5}+20 x^{3}+120 x-120 \sin x\right) \\
& +\frac{\hbar}{120}\left(\frac{\hbar x^{5}+20 \hbar x^{3}+20 x^{3}}{s}\right)
\end{aligned}
$$

In the same manner, the rest of components were obtained using the Matlab package.

Taking the inverse Laplace of components yields

$$
\begin{aligned}
u_{1}(x, t)= & x+\cosh t \\
u_{2}(x, t)= & \frac{h}{6}\left(x^{3}+\left(6 x-6 \sin x-x^{3}\right) \delta(1, t)\right) \\
u_{3}(x, t)= & \frac{h^{2}}{120}\left(x^{5}+\left(120 x-120 \sin x+x^{5}-20 x^{3}\right) \delta(3, t)\right) \\
& +\frac{h}{60}\left(10 x^{3}(1+\hbar)+\left(60 x-60 \sin x-10 x^{3}-\hbar x^{5}\right) \delta(1, t)\right)
\end{aligned}
$$

Finally, the $M$ th-order approximation solution of Eq. (3.17) is as follows

$$
S_{M}=\sum_{k=0}^{M} u_{k}(x, t) .
$$

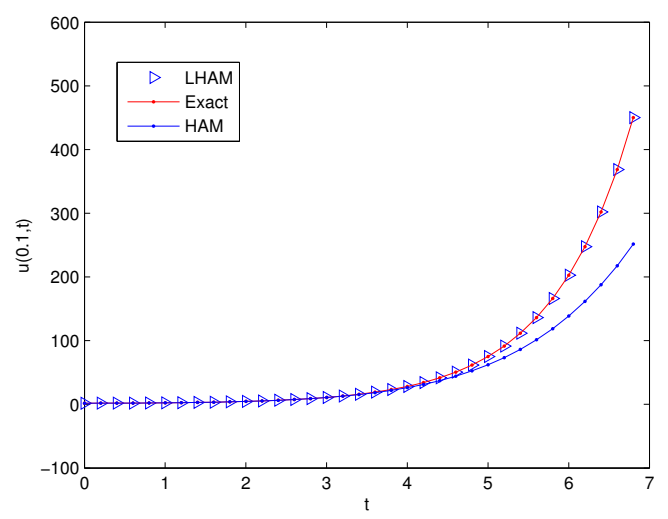

Figure 1: Comparision between LHAM, HAM and exact solution for $x=0.1$.

Table 2 describes the analytic 4th-order approximation solution of Eq. (3.17) by the LHAM with $\hbar=-1$ and the analytic 11th-order approximation solution of Eq. (3.17) by the HAM with different 
value of $\hbar$ when $x=0.1$ and $1 \leq t \leq 7$. Table 2 shows that, unlike the LHPM, the absolute error of the HAM is dramatically increased as the time value $t$ increases, so the HAM solution validity range is restricted to a short region $(t<2)$. But the validity range can be increased by increasing the terms of the solution to more than 11 terms. On the other hand, results of the LHAM are in good agreement with those obtained by exact solution. The LHAM solution is almost valid for a large wide range of times that shows that the present method can solve a non-homogeneous parabolic equation with a high degree of accuracy by the four first terms only.

Table 2: The absolute error of LHAM and HAM with $x=0.1$

\begin{tabular}{cccc}
\hline$t$ & $L H A M(\hbar=-1)$ & $H A M(\hbar=-0.9)$ & $H A M(\hbar=-1)$ \\
\hline 1 & $2.6645 \times 10^{-15}$ & $1.4785 \times 10^{-10}$ & 0 \\
2 & $3.1086 \times 10^{-15}$ & $2.2231 \times 10^{-8}$ & 0 \\
3 & $3.5527 \times 10^{-15}$ & $1.1438 \times 10^{-6}$ & $4.6185 \times 10^{-13}$ \\
4 & $3.5527 \times 10^{-15}$ & $3.2658 \times 10^{-5}$ & $4.6507 \times 10^{-10}$ \\
5 & $4.4211 \times 10^{-14}$ & $5.5076 \times 10^{-4}$ & $9.9888 \times 10^{-8}$ \\
6 & $2.8422 \times 10^{-14}$ & $8.7163 \times 10^{-3}$ & $8.0810 \times 10^{-6}$ \\
7 & 0 & $9.6781 \times 10^{-2}$ & $3.3365 \times 10^{-4}$ \\
\hline
\end{tabular}

Example 3.4. Let us consider the one dimensional non-homogeneous problem

$$
\frac{\partial u}{\partial t}=\frac{\partial^{2} u}{\partial x^{2}}+e^{-x}(\cos x-\sin x)
$$

subject to boundary conditions

$$
u(0, t)=\sin t, \quad u_{x}(0, t)=1-\sin t,
$$

and the initial condition $u(x, 0)=x$ that is easily seen to have the exact solution $u(x, t)=x+e^{-x} \sin t$.

\section{HAM approach}

To solve Eq. (3.27) by means of HAM, we choose the linear operator

$$
\mathcal{L}[\phi(x, t ; p)]=\frac{\partial \phi(x, t ; p)}{\partial t} .
$$

Furthermore, Eq. (3.27) suggests to define the nonlinear operator

$$
\mathcal{N}[\phi(x, t ; p)]=\frac{\partial \phi(x, t ; p)}{\partial t}-\frac{\partial^{2} \phi(x, t ; p)}{\partial x^{2}}-e^{-x}(\cos x-\sin x) .
$$

Using above definition, we construct the zeroth-order deformation equation

$$
(1-p) \mathcal{L}\left[\phi(x, t ; p)-u_{0}(x, t)\right]=p \hbar \mathcal{H}(x, t) \mathcal{N}[\phi(x, t ; p)] .
$$

According to Eqs. (3.28), (3.29), we gain the $m$ th-order $(m \geq 1)$ deformation equation

$$
\mathcal{L}\left[u_{m}(x, t)-\chi_{m} u_{m-1}(x, t)\right]=\hbar \mathcal{H}(x, t) \mathcal{R}_{m}\left(\vec{u}_{m-1}\right),
$$

where

$$
\mathcal{R}_{m}\left(\vec{u}_{m-1}\right)=\frac{\partial u_{m-1}}{\partial t}+\frac{\partial^{2} u_{m-1}}{\partial x^{2}}-\left(1-\chi_{m}\right) e^{-x}(\cos x-\sin x) .
$$


Now, the components of solution of Eq. (3.27) for $(m \geq 1)$ becomes

$$
u_{m}(x, t)=\chi_{m} u_{m-1}(x, t)+\hbar \int_{0}^{t} \mathcal{H}(x, \xi) \mathcal{R}_{m}\left(\vec{u}_{m-1}(x, \xi)\right) d \xi
$$

The initial approximation $u_{0}(x, t)$ can be freely chosen, here we set

$$
u_{0}(x, t)=x .
$$

Then, the solution of Eq. (3.27) in series form is given by

$$
u(x, t)=u_{0}(x, t)+u_{1}(x, t)+\cdots
$$

\section{LHAM approach}

By applying a Laplace transform on both sides of Eqs. (3.27), (3.28) and in view of the initial condition, we get

$$
\begin{aligned}
\frac{\partial^{2} \bar{u}}{\partial x^{2}}-s \bar{u}+x+e^{-x}\left(\frac{s-1}{s^{2}+1}\right) & =0 \\
\bar{u}(0, s)=\frac{1}{s^{2}+1}, & \bar{u}_{x}(0, s)=\frac{1}{s}-\frac{1}{s^{2}+1} .
\end{aligned}
$$

Now, we solve Eq. (3.31) by means of HAM. Therefore, we choose the linear oprator

$$
\mathcal{L}[\phi(x, s ; p)]=\frac{\partial^{2} \phi(x, s ; p)}{\partial x^{2}}
$$

We define a nonlinear operator as

$$
\mathcal{N}[\phi(x, s ; p)]=\frac{\partial^{2} \phi(x, s ; p)}{\partial x^{2}}-s \phi(x, s ; p)+x+e^{-x}\left(\frac{s-1}{s^{2}+1}\right) .
$$

Using the definition (3.33) and (3.34), we construct the zeroth-order deformation equation

$$
(1-p) \mathcal{L}\left[\phi(x, s ; p)-\bar{u}_{0}(x, s)\right]=p \hbar \mathcal{H}(x, s) \mathcal{N}[\phi(x, s ; p)] .
$$

Thus, we obtain the $m$ th-order $(m \geq 1)$ deformation equation

$$
\mathcal{L}\left[\bar{u}_{m}(x, s)-\chi_{m} \bar{u}_{m-1}(x, s)\right]=\hbar \mathcal{H}(x, s) \mathcal{R}_{m}\left(\overrightarrow{\bar{u}}_{m-1}\right),
$$

where

$$
\mathcal{R}_{m}\left(\overrightarrow{\bar{u}}_{m-1}\right)=\frac{\partial^{2} \bar{u}_{m-1}}{\partial x^{2}}-\bar{u}_{m-1}+\left(1-\chi_{m}\right)\left(x+e^{-x}\left(\frac{s-1}{s^{2}+1}\right)\right) .
$$

Now, the solution of the $m$ th-order $(m \geq 1)$ deformation equation $(3.26)$ is as follows

$$
\bar{u}_{m}(x, s)=\chi_{m} \bar{u}_{m-1}(x, s)+\hbar \int_{0}^{x} \int_{0}^{x} \mathcal{H}(\xi, s) \mathcal{R}_{m}\left(\overrightarrow{\bar{u}}_{m-1}(\xi, s)\right) d \xi d \xi .
$$

The initial approximation $\bar{u}_{0}(x, s)$ can be freely chosen, here we set

$$
\bar{u}_{0}(x, s)=\frac{1-x}{s^{2}+1}+\frac{x}{s}
$$


which satisfies the boundary conditions 3.32 . By choosing $H(x, s)=1$, the components of the LHAM solution for Eq. (3.31) are derived as follows

$$
\bar{u}_{1}(x, s)=\frac{\hbar}{6}\left(\frac{-6 s+6+6 s x-6 x-3 s x^{2}+s x^{3}+6 s e^{-x}}{s^{2}+1}\right),
$$

In the same manner, the rest of components were obtained using the Matlab package.

Taking the inverse Laplace of components yields

$$
\begin{aligned}
u_{0}(x, t) & =x-(x-1) \sin t \\
u_{1}(x, t)= & \frac{h}{6}\left(\left(-6+6 x-3 x^{2}+x^{3}+6 e^{-x}\right) \cos t\right) \\
& +\frac{h}{6}\left(\left(6-6 x-6 e^{-x}\right) \sin t\right)
\end{aligned}
$$

Finally, the $M$ th-order approximation solution of Eq. (3.27) is as follows

$$
S_{M}=\sum_{k=0}^{M} u_{k}(x, t) .
$$
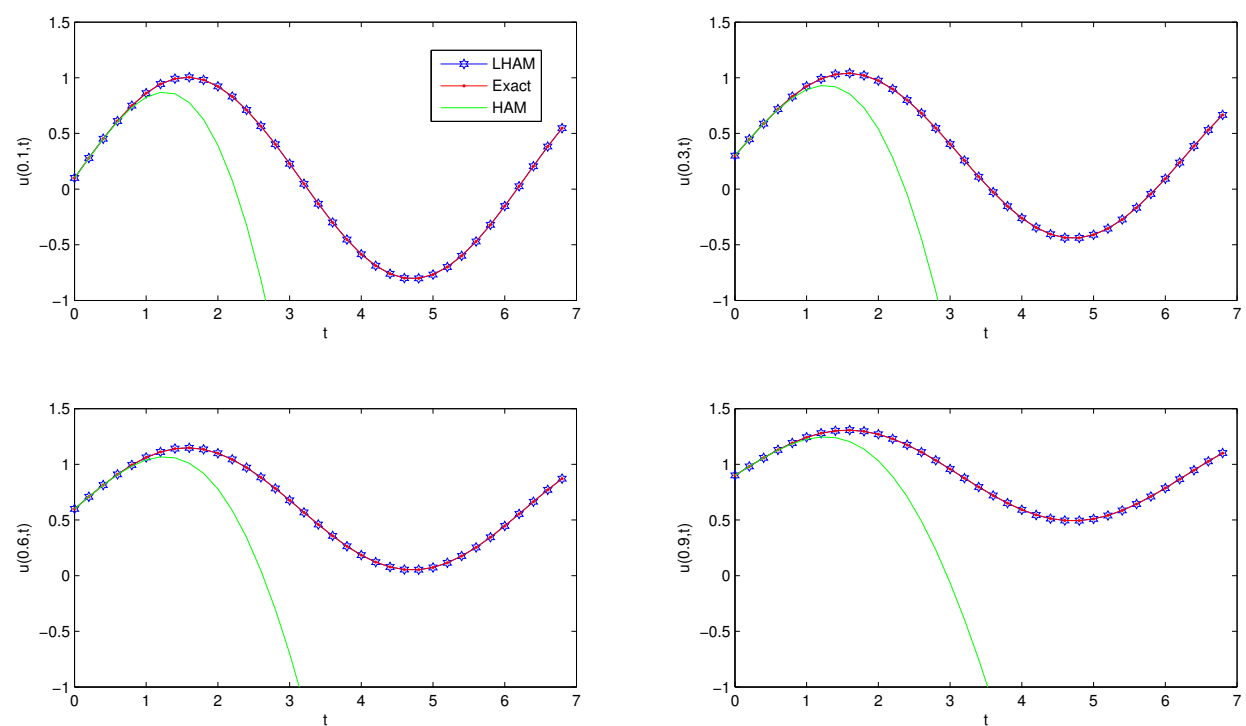

Figure 2: Comparision between LHAM, HAM and exact solution for $x=0.1, x=0.3, x=0.6, x=0.9$.

Table 3 describes the analytic 5th-order approximation solution of Eq. (3.27) by the LHAM with $\hbar=-1$ and the analytic 10th-order approximation solution of Eq. (3.27) by the HAM with different values of $\hbar$ when $x=0.1$ and $1 \leq t \leq 7$. 
Table 3: The absolute error of LHAM and HAM with $x=0.1$

\begin{tabular}{cccc}
\hline$t$ & $\operatorname{LHAM}(\hbar=-1)$ & $H A M(\hbar=-1.1)$ & $H A M(\hbar=-1)$ \\
\hline 1 & $1.1102 \times 10^{-16}$ & $3.6750 \times 10^{-10}$ & $2.2523 \times 10^{-8}$ \\
2 & 0 & $8.0508 \times 10^{-8}$ & $4.5256 \times 10^{-5}$ \\
3 & 0 & $1.5224 \times 10^{-5}$ & $3.7935 \times 10^{-3}$ \\
4 & 0 & $7.1558 \times 10^{-3}$ & $8.6027 \times 10^{-2}$ \\
5 & 0 & $2.5672 \times 10^{-1}$ & $9.4877 \times 10^{-1}$ \\
6 & 0 & $2.4667 \times 10^{+0}$ & $6.6125 \times 10^{+0}$ \\
7 & $1.1102 \times 10^{-16}$ & $1.7738 \times 10^{+1}$ & $3.3512 \times 10^{+1}$ \\
\hline
\end{tabular}

\section{Conclusion}

In this paper, the LHAM and HAM were used to obtain the analytic solutions of linear and non-linear partial differential equations. The comparison between the LHAM and HAM were made and it was found that LHAM is more effective than HAM. On the other hand, the LHAM solution is almost valid for a large wide range of times that shows that the present method can solve a nonhomogeneous parabolic equation with a high degree of accuracy. Hence, it may be concluded that this method is a powerful and an efficient technique in finding the analytic solutions for wide classes of problems. The computations associated with the example in this paper were performed using Matlab 7.

\section{References}

[1] G. Adomian, Solving Frontier Problems of Physics: The Decomposition Method, Fundamental Theories of Physics, 60. Kluwer Academic Publishers Group, Dordrecht, (1994). 1

[2] A. K. Alomari, A novel solution for fractional chaotic Chen system, J. Nonlinear Sci. Appl., 8 (2015), 478-488.

[3] J. Biazar, M. Gholami Porshokuhi, B. Ghanbari, Extracting a general iterative method from an adomian decomposition method and comparing it to the variational iteration method, Comput. Math. Appl., 59 (2010), 622-628. 1. 2

[4] C. Chun, Fourier-series-based variational iteration method for a reliable treatment of heat equations with variable coefficients, Int. J. Nonlinear Sci. Numer. Simul., 10 (2009), 1383-1388. 1

[5] N. Faraz, Y. Khan, A. Yildirim, Analytical approach to two-dimensional viscous flow with a shrinking sheet via variational iteration algorithm-II, J. King Saud University, 1 (2011), 77-81.1

[6] J. H. He, Homotopy perturbation technique, Comput. Methods Appl. Mech. Eng., 178 (1999), 257-262. 1

[7] J. H. He, Variational iteration method-a kind of nonlinear analytical technique: some examples, Int. J. Nonlinear Mech., 34 (1999), 699-708.1

[8] J. H. He, X. H. Wu, Variational iteration method: new development and applications, Comput. Math. Appl., 54 (2007), 881-894.1.

[9] J. H. He, G. C. Wu, F. Austin, The variational iteration method which should be followed, Nonlinear Sci. Lett. A, 1 (2010), 1-30. 1

[10] E. Hesameddini, H. Latifizadeh, Reconstruction of variational iteration algorithms using the Laplace transform, Int. J. Nonlinear Sci. Numer. Simul., 10 (2009), 1377-1382.1.

[11] R. Hirota, Exact solutions of the Korteweg-de Vries equation for multiple collisions of solitons, Phys. Rev. Lett., 27 (1971), 1192-1194.1.

[12] S. Islam, Y. Khan, N. Faraz, F. Austin, Numerical solution of logistic differential equations by using the Laplace decomposition method, World Appl. Sci. J., 8 (2010), 1100-1105. 1

[13] H. Jafari, C. Chun, S. Seifi, M. Saeidy, Analytical solution for nonlinear Gas Dynamic equation by Homotopy Analysis Method, Appl. Appl. Math., 4 (2009), 149-154. 1 
[14] H. Jafari, M. Saeidy, M. A. Firoozjaee, The Homotopy analysis method for solving higher dimensional initial boundary value problems of variable cofficients, Numer. Meth. Part. D. E., 26 (2010), 1021-1032. 1

[15] A. V. Karmishin, A. I. Zhukov, V. G. Kolosov, Methods of dynamics calculation and testing for thinwalled structures, Mashinostroyenie, Moscow, Russia, (1990). 1

[16] S. A. Khuri, A Laplace decomposition algorithm applied to a class of nonlinear differential equations, J. Appl. Math., 1 (2001), 141-155. 1

[17] Y. Khan, An effective modification of the Laplace decomposition method for nonlinear equations, Int. J. Nonlinear Sci. Numer. Simul., 10 (2009), 1373-1376.1

[18] Y. Khan, N. Faraz, A new approach to differential difference equations, J. Adv. Res. Differ. Equations, $2(2010), 1-12.1$

[19] S. J. Liao, The proposed homotopy analysis technique for the solution of nonlinear problems, Ph.D. Thesis, Shanghai Jiao Tong University, (1992). 1

[20] S. J. Liao, An approximate solution technique which does not depend upon small parameters (Part 2): an application in fluid mechanics, Int. J. Nonlinear Mech., 32 (1997), 815-822.1, 3

[21] S. J. Liao, Beyond perturbation: introduction to the homotopy analysis method, CRC Press, Boca Raton: Chapman Hall, (2003).11

[22] A. M. Lyapunov, The general problem of the stability of motion, (English translation), Taylor \& Francis, London, UK, (1992).1, 2, 2,

[23] J. Saberi Nadjafi, A. Ghorbani, He's homotopy perturbation method: an effective tool for solving nonlinear integral and integro-differential equations, Comput. Math. Appl., 58 (2009), 2379-2390.1

[24] L. A. Soltani, A. Shirzadi, A new modification of the variational iteration method, Comput. Math. Appl., 59 (2010), 2528-2535.1

[25] A. M. Wazwaz, On multiple soliton solutions for coupled KdV-mkdV equation, Nonlinear Sci. Lett. A, 1 (2010), 289-296.1.

[26] G. C. Wu, J. H. He, Fractional calculus of variations in fractal spacetime, Nonlinear Sci. Lett. A, 1 (2010), 281-287.1

[27] G. C. Wu, E. W. M. Lee, Fractional variational iteration method and its application, Phys. Lett. A, 374 (2010), 2506-2509. 1

[28] Z. Yao, Almost periodicity of impulsive Hematopoiesis model with infinite delay, J. Nonlinear Sci. Appl., 8 (2015), 856-865.

[29] E. Yusufoglu, Numerical solution of Duffng equation by the Laplace decomposition algorithm, Appl. Math. Comput., 177 (2006), 572-580. 1 